\title{
THE EXISTENCE OF A COADJOINT EQUIVARIANT MOMENTUM MAPPING FOR A SEMIDIRECT PRODUCT
}

\author{
KENTARO MIKAMI
}

\begin{abstract}
We consider a symplectic action of a group $G$ on a symplectic manifold $P$, which admits a momentum mapping. Assume that $G$ is a semidirect product of $G_{1}$ by $G_{2}$. We prove that if the symplectic action of $G_{1}$ has a coadjoint equivariant momentum mapping, and if $H^{1}\left(g_{1}: \mathbf{R}\right)=H^{2}\left(g_{2}: \mathbf{R}\right)=0$, then the symplectic action of $G$ has a coadjoint equivariant momentum mapping, where $g_{1}$ and $g_{2}$ are the Lie algebras of $G_{1}$ and $G_{2}$ respectively.
\end{abstract}

1. Introduction and statement of result. Let $(P, \omega)$ be a finite dimensional connected symplectic manifold, that is, $P$ is a finite dimensional connected smooth manifold with a nondegenerate closed 2 -form $\omega$.

Let $G$ be a finite dimensional Lie group and $\Phi: G \times P \rightarrow P$ be a symplectic action of $G$ on $P$, that is, for each $g \in G$ the map

$$
\Phi_{g}: P \rightarrow P, \quad x \mapsto \Phi(g, x)
$$

is symplectic, that is, $\Phi_{g}^{*} \omega=\omega$.

J.-M. Souriau defined momentum mappings for the symplectic action, which are the group theoretical analogue of the linear and angular momentum associated with the translational and rotational invariance. The importance of momentum mappings in mechanics can easily be seen. For example, let $f$ be a Hamiltonian, and assume that $f$ is $(G, \Phi)$-invariant, that is, for each $g \in G, \Phi_{g}^{*} f=f$ holds. Then the momentum mapping is constant along the integral curves of the Hamiltonian vector field of $f$.

Not every symplectic action has a momentum mapping (cf. [5, p. 18]), and we have some exotic examples where momentum mappings are not coadjoint equivariant (cf. [5, p. 19]). On the other hand, we find some conditions which guarantee the existence of a coadjoint equivariant momentum mapping. For example, if the symplectic form is an exact form of a $(G, \Phi)$-invariant 1-form (that is a case which we encounter frequently in mechanics) or if $G$ is connected and semisimple (only if $\left.H^{1}(g: \mathbf{R})=H^{2}(g: \mathbf{R})=0\right)$, then the symplectic action $(G, \Phi)$ has a coadjoint equivariant momentum mapping (cf. [1], [4]). Under some suitable global conditions, a (coadjoint equivariant) momentum mapping supplies a reduced phase space, where we can eliminate some of the variables of the Hamiltonian system (cf. [1], [2]). G.-M. Marle [2] proved that if the symplectic action $(G, \Phi)$ has a

Received by the editors May 18, 1980 and, in revised form, November 3, 1980.

1980 Mathematics Subject Classification. Primary 22 E70.

Key words and phrases. Symplectic action, coajoint equivariant momentum mapping, semidirect product. 
momentum mapping and the group $G$ is a semidirect product of closed subgroups $G_{\mu}$ and $H$, then the reduced phase space $Q$ of Marle is symplecto-diffeomorphic to the product of symplectic manifolds $J^{-1}(\mu) / G_{\mu}$ and $G \cdot \mu$, where $G_{\mu}$ and $G \cdot \mu$ are the isotropy group at $\mu$ and the orbit through $\mu$ of the left affine action on $g^{*}$ determined by the momentum mapping. If the momentum mapping is coadjoint equivariant, then this action is just the coadjoint action, and the isotropy group $G_{\mu}$ and the orbit $G \cdot \mu$ are the familiar ones. And we have that a Lie algebra which is neither simple nor 1-dimensional abelian, is a semidirect product of two Lie algebras of smaller dimension. So we consider the problem of the existence of coadjoint equivariant momentum mappings when the group $G$ is a semidirect product, that is, $G=G_{1} \times{ }_{\sigma} G_{2}$ where $\sigma$ is a homomorphism of $G_{2}$ into the automorphism group $\operatorname{Aut}\left(G_{1}\right)$ of $G_{1}$. Let $\Phi^{(i)}$ be the restriction of $\Phi$ to the subgroup $G_{i}(i=1,2)$. If $(G, \Phi)$ is a symplectic action, then $\left(G_{i}, \Phi^{(i)}\right)$ is also a symplectic action $(i=1,2)$.

Our result is the following

TheOREM. Let $(P, \omega)$ be a connected symplectic manifold and $(G, \Phi)$ be a symplectic action on $(P, \omega)$ admitting a momentum mapping. Assume that $G$ is connected and $a$ semidirect product of $G_{1}$ by $G_{2}$ determined by $\sigma$, that is, $G=G_{1} \times{ }_{0} G_{2}$ where $\sigma \in \operatorname{Hom}\left(G_{2}, \operatorname{Aut}\left(G_{1}\right)\right)$. If $\left(G_{1}, \Phi^{(1)}\right)$ has a coadjoint equivariant momentum mapping and $H^{1}\left(g_{1}: \mathbf{R}\right)=H^{2}\left(g_{2}: \mathbf{R}\right)=0$, then $(G, \Phi)$ has a coadjoint equivariant momentum mapping.

2. Preliminaries. In the exact sequence of Lie algebra homomorphisms

$$
0 \rightarrow \mathbf{R} \hookrightarrow \mathscr{F}(P) \stackrel{\beta}{\rightarrow} \mathscr{X}(P: \omega) \stackrel{\gamma}{\rightarrow} H^{1}(P: \mathbf{R}) \rightarrow 0,
$$

$\mathscr{F}(P)$ is the space of all $\mathbf{R}$-valued smooth functions on $P . \mathscr{X}(P: \omega)$ is the Lie algebra of all smooth vector fields $X$ on $P$ satisfying $L_{X} \omega=0$, where $L_{X}$ is the Lie differentiation with respect to $X . \beta$ is a mapping defined by

$$
\beta(f)\lrcorner \omega=-d f \text { for each } f \in \mathcal{F}(P),
$$

where $\beta(f)\lrcorner \omega$ is the inner product of the vector field $\beta(f)$ and $\omega$. For each $f, h$ in $\mathscr{F}(P)$ we define the Poisson bracket $\{f, h\}$ by

$$
\{f, h\}=\omega(\beta(f), \beta(h)) .
$$

Then $\mathscr{F}(P)$ becomes a Lie algebra with respect to the Poisson bracket, and $\beta$ is a Lie algebra homomorphism of $\mathscr{F}(P)$ into $\mathscr{X}(P: \omega) . \beta(f)$ is called the Hamiltonian vector field of the Hamiltonian $f$. For each $X$ in $\mathcal{X}(P: \omega)$ we define $\gamma(X)$ by the de Rham cohomology class of $X\lrcorner \omega$. Since $\gamma([X, Y])=0$ for each $X, Y$ in $\mathcal{X}(P: \omega)$, let us introduce in the first cohomology group $H^{1}(P: \mathbf{R})$ the trivial Lie algebra structure. Then $\gamma$ is a Lie algebra homomorphism of $\mathscr{X}(P: \omega)$ into $H^{1}(P: \mathbf{R})$, and the above sequence is exact.

Let $(G, \Phi)$ be a left action on $P$ and $g$ be the Lie algebra of $G$. For each $\xi \in \mathcal{g}$, we mean by $\rho(\xi)$ the fundamental vector field on $\boldsymbol{P}$ corresponding to $-\xi$,

$$
\rho(\xi)=\frac{d}{d t} \Phi_{\exp -\left.t \xi\right|_{t \rightarrow 0}}
$$


Then $\rho$ is a Lie algebra homomorphism of $\mathcal{g}$ into the algebra of all vector fields on $P$. In particular, if $(G, \Phi)$ is a symplectic action, then $\operatorname{Im}(\rho) \subset \mathcal{X}(P: \omega)$, and $\rho$ is a Lie algebra homomorphism of $\mathscr{G}$ into $\mathscr{X}(P: \omega)$.

Definition 1. A mapping $J: P \rightarrow g^{*}$ (= the dual space of the Lie algebra $\mathscr{g}$ of $G)$ is called a momentum mapping for the symplectic action $(G, \Phi)$, provided that for every $\xi \in \mathcal{G}$

$$
d[\hat{J}(\xi)]=-\rho(\xi)\lrcorner \omega,
$$

where $\hat{J}(\xi): P \rightarrow \mathbf{R}$ is defined by $\hat{J}(\xi)(x)=\langle\xi, J(x)\rangle$.

This Definition 1 is equivalent to $\hat{J}: \mathcal{G} \rightarrow \mathscr{F}(P)$, the linear mapping satisfying $\beta \circ \hat{J}=\rho$. We call such $\hat{J}$ a linear lift of $\rho$.

Definition 2. A momentum mapping $J$ for the symplectic action $(G, \Phi)$ is called

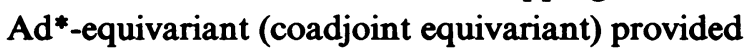

$$
J \circ \Phi_{g}=\operatorname{Ad}_{g^{-1}}^{*} \circ J \quad \text { for every } g \in G .
$$

The equation in Definition 2 is equivalent to

$$
\hat{J}\left(\operatorname{Ad}_{g^{-1}} \eta\right)=\hat{J}(\eta) \circ \Phi_{g} \quad \text { for every } g \in G \text { and } \eta \in g .
$$

Differentiating the above equation in $g$, we have that $\hat{J}$ is a Lie algebra homomorphism of $\mathcal{G}$ into $\mathscr{F}(P)$. The converse is also true if $G$ is connected, that is, we have the following lemma which makes it possible for us to prove our theorem in the Lie algebra category instead of the group category.

LemMA. For a symplectic action $(G, \Phi)$ on a connected symplectic manifold $(P, \omega)$, assume that there is a Lie algebra homomorphic lift $\lambda$ of $\rho$, that is, $\lambda$ is a Lie algebra homomorphism of $\mathcal{G}$ into $\mathscr{F}(P)$ satisfying $\beta \circ \lambda=\rho$. If $G$ is connected, then $(G, \Phi)$ has an $\mathrm{Ad}^{*}$-equivariant momentum mapping $J$ defined by $\hat{J}=\lambda$.

Proof. For each $g \in G$ and $\xi \in \mathcal{g}$

$$
\beta\left(\Phi_{g}^{*} \lambda(\xi)\right)=\rho\left(\operatorname{Ad}_{g^{-1}} \xi\right)
$$

Take any $\eta \in \mathcal{G}$ and fix it. We consider a mapping $\psi: G \times P \rightarrow \mathbf{R}$ defined by

$$
\psi(g, x)=\left(\Phi_{g}^{*} \lambda(\eta)\right)(x)-\lambda\left(\operatorname{Ad}_{g^{-1}} \eta\right)(x)
$$

for each $g \in G$ and $x \in P . \psi$ is an expression of the obstruction of Ad*-equivariance of $J$ defined by $\hat{J}=\lambda$. By using the relation (*) we have

$$
\begin{aligned}
\beta \circ \psi_{g} & =\beta\left(\Phi_{g}^{*} \lambda(\eta)\right)-\beta \circ \lambda\left(\operatorname{Ad}_{g^{-1}} \eta\right) \\
& =\rho\left(\operatorname{Ad}_{g^{-1} \eta}\right)-\rho\left(\operatorname{Ad}_{g^{-1}} \eta\right) \\
& =0
\end{aligned}
$$


where $\psi_{g}: P \rightarrow \mathbf{R}, x \mapsto \psi_{g}(x)=\psi(g, x)$. This implies that $\psi(g, x)$ is independent of $x$. For each $g \in G$ and $\xi \in \dot{q}$, we have

$$
\begin{aligned}
\left.\frac{d}{d t} \psi(g \exp t \xi, x)\right|_{t=0} & =\left.d[\lambda(\eta)]\left(\rho\left(\operatorname{Ad}_{g} \xi\right)\right)\right|_{\Phi(g, x)}+\lambda\left(\operatorname{ad}_{\xi} \operatorname{Ad}_{g^{-1}} \eta\right)(x) \\
& =\Phi_{g}^{*}\left\{\lambda(\eta), \lambda\left(\operatorname{Ad}_{g} \xi\right)\right\}(x)+\lambda\left(\operatorname{ad}_{\xi} \operatorname{Ad}_{g^{-1}} \eta\right)(x) \\
& =\left\{\Phi_{g}^{*} \lambda(\eta), \Phi_{g}^{*} \lambda\left(\operatorname{Ad}_{g} \xi\right)\right\}(x)+\lambda\left(\operatorname{ad}_{\xi} \operatorname{Ad}_{g^{-1} \eta}\right)(x) \\
& =\left\{\lambda\left(\operatorname{Ad}_{g^{-1}} \eta\right), \lambda(\xi)\right\}(x)+\lambda\left(\left[\xi, \operatorname{Ad}_{g^{-1}} \eta\right]\right)(x) \\
& =0
\end{aligned}
$$

because $\lambda$ is a Lie algebra homomorphism. Therefore $\psi(g, x)$ is constant on each connected component of $G \times P$. Since $G$ is connected, $\psi(e, x)=0$ implies that $\psi=0$ identically. Thus we complete the proof of the lemma.

3. Proof of the theorem. The group operation of $G=G_{1} \times{ }_{\sigma} G_{2}$ where $\sigma \in$ $\operatorname{Hom}\left(G_{2}, \operatorname{Aut}\left(G_{1}\right)\right)$ is as follows:

$$
\left(g_{1}, g_{2}\right)\left(h_{1}, h_{2}\right)=\left(g_{1} \sigma_{g_{2}}\left(h_{1}\right), g_{2} h_{2}\right)
$$

As a vector space, the Lie algebra $\mathcal{g}$ of $G=G_{1} \times{ }_{\sigma} G_{2}$ is a direct product of $g_{1}$ and $g_{2}$, and the Lie algebra structure is as follows:

$$
\left[\left(\xi_{1}, \xi_{2}\right),\left(\eta_{1}, \eta_{2}\right)\right]=\left(\left[\xi_{1}, \eta_{1}\right]+\dot{\sigma}_{\xi_{2}} \eta_{1}-\circ_{\eta_{2}} \xi_{1},\left[\xi_{2}, \eta_{2}\right]\right) \text {, }
$$

where $\xi_{1}, \eta_{1} \in g_{1}, \xi_{2}, \eta_{2} \in g_{2}$ and $\%$ is a Lie algebra homomorphism of $g_{2}$ into the algebra of derivations of $g_{1}$ determined by $\sigma$. Let $\rho, \rho_{1}$ and $\rho_{2}$ be the maps assigning the fundamental vector fields of the actions $(G, \Phi),\left(G_{1}, \Phi^{(1)}\right)$ and $\left(G_{2}, \Phi^{(2)}\right)$ respectively. Then $\rho\left(\xi_{1}, \xi_{2}\right)=\rho_{1}\left(\xi_{1}\right)+\rho_{2}\left(\xi_{2}\right)$ for each $\left(\xi_{1}, \xi_{2}\right) \in g=g_{1} \times g_{2}$. Since the symplectic action $(G, \Phi)$ has a momentum mapping $J, \hat{J}$ is a linear lift of $\rho$. Let $\lambda_{i}$ be the restriction of $\hat{J}$ to $g_{i}(i=1,2)$. Then $\lambda_{i}$ is a linear lift of $\rho_{i}(i=1,2)$. So $\lambda_{i}$ defines a momentum mapping for $\left(G_{i}, \Phi^{(i)}\right)(i=1,2)$. Since $\left(G_{1}, \Phi^{(1)}\right)$ has an Ad*-equivariant momentum mapping, there is a $\mu_{1} \in g_{1}^{*}$ such that $\lambda_{1}+\mu_{1}$ is a Lie algebra homomorphic lift of $\rho_{1}$. Since $\left(\lambda_{1}+\mu_{1}\right)+\lambda_{2}$ is also a linear lift of $\rho$, we may assume in the first decomposition $\hat{J}=\lambda_{1}+\lambda_{2}$ that $\lambda_{1}$ is a Lie algebra homomorphic lift of $\rho_{1}$. Define a mapping $\nu_{2}$ of $g_{2} \times g_{2}$ into $\mathscr{F}(P)$ by

$$
\nu_{2}(\xi, \eta)=\left\{\lambda_{2}(\xi), \lambda_{2}(\eta)\right\}-\lambda_{2}([\xi, \eta])
$$

for each $\xi, \eta$ in $g_{2}$. Since $\beta \circ \nu_{2}=0, \nu_{2}$ is a bilinear mapping of $g_{2} \times g_{2}$ into $\mathbf{R}$, and so $\nu_{2}$ is a 2-dimensional cochain of $g_{2}$. Using the Jacobi identities of Lie and Poisson, it is easily proved that $\nu_{2}$ is closed. The condition $H^{2}\left(g_{2}: \mathbf{R}\right)=0$ implies $\nu_{2}=\delta \mu_{2}$ for some $\mu_{2} \in g_{2}^{*}$, where $\delta$ is the coboundary operator of $g_{2}$. We can easily see that $\lambda_{2}-\mu_{2}$ is a Lie algebra homomorphic lift of $\rho_{2}$. So we may assume that the linear lift $\hat{J}$ is the sum of Lie algebra homomorphic lifts $\lambda_{1}$ and $\lambda_{2}$ which correspond to $\rho_{1}$ and $\rho_{2}$ respectively. To show that $\hat{J}$ is a Lie algebra homomorphism, we consider a mapping $\nu$ of $\mathscr{g} \times \mathcal{G}$ into $\mathscr{F}(P)$ defined by

$$
\nu\left(\left(\xi_{1}, \xi_{2}\right),\left(\eta_{1}, \eta_{2}\right)\right)=\left\{\hat{J}\left(\xi_{1}, \xi_{2}\right), \hat{J}\left(\eta_{1}, \eta_{2}\right)\right\}-\hat{J}\left(\left[\left(\xi_{1}, \xi_{2}\right),\left(\eta_{1}, \eta_{2}\right)\right]\right),
$$

where $\left(\xi_{1}, \xi_{2}\right),\left(\eta_{1}, \eta_{2}\right) \in g_{1} \times g_{2}=g$. Using the fact that $\lambda_{1}$ and $\lambda_{2}$ are Lie algebra 
homomorphisms, we have

$$
\begin{aligned}
\nu\left(\left(\xi_{1}, \xi_{2}\right),\left(\eta_{1}, \eta_{2}\right)\right)= & \left\{\lambda_{1}\left(\xi_{1}\right), \lambda_{2}\left(\eta_{2}\right)\right\}+\lambda_{1}\left(\dot{\sigma}_{\eta_{2}} \xi_{1}\right) \\
& -\left\{\lambda_{1}\left(\eta_{1}\right), \lambda_{2}\left(\xi_{2}\right)\right\}-\lambda_{1}\left(\dot{\circ}_{\xi_{2}} \eta_{1}\right) .
\end{aligned}
$$

Since we have, for each $\xi \in g_{1}$ and $\eta \in g_{2}$

$$
\begin{aligned}
\beta\left[\left\{\lambda_{1}(\xi), \lambda_{2}(\eta)\right\}+\lambda_{1}\left(\dot{\sigma}_{\eta} \xi\right)\right] & =\left[\beta \circ \lambda_{1}(\xi), \beta \circ \lambda_{2}(\eta)\right]+\beta \circ \lambda_{1}\left(\dot{\sigma}_{\eta} \xi\right) \\
& =\left[\rho_{1}(\xi), \rho_{2}(\eta)\right]+\rho_{1}\left(\dot{\sigma}_{\eta} \xi\right) \\
& =\rho([(\xi, 0),(0, \eta)])+\rho_{1}\left(\dot{\sigma}_{\eta} \xi\right) \\
& =-\rho_{1}\left(\dot{\sigma}_{\eta} \xi\right)+\rho_{1}\left(\dot{\sigma}_{\eta} \xi\right) \\
& =0,
\end{aligned}
$$

$\left\{\lambda_{1}(\xi), \lambda_{2}(\eta)\right\}+\lambda_{1}\left(\circ_{\eta} \xi\right)$ is constant, which we denote by $c(\xi, \eta)$. Now we have $\nu\left(\left(\xi_{1}, \xi_{2}\right),\left(\eta_{1}, \eta_{2}\right)\right)=c\left(\xi_{1}, \eta_{2}\right)-c\left(\eta_{1}, \xi_{2}\right)$. For each $\xi, \xi^{\prime} \in g_{1}$ and $\eta \in g_{2}$, we have

$$
\begin{aligned}
c\left(\left[\xi, \xi^{\prime}\right], \eta\right)= & \left\{\lambda_{1}\left(\left[\xi, \xi^{\prime}\right]\right), \lambda_{2}(\eta)\right\}+\lambda_{1}\left(\circ_{\eta}\left[\xi, \xi^{\prime}\right]\right) \\
= & \left\{\left\{\lambda_{1}(\xi), \lambda_{1}\left(\xi^{\prime}\right)\right\}, \lambda_{2}(\eta)\right\}+\lambda_{1}\left(\circ_{\eta}\left[\xi, \xi^{\prime}\right]\right) \\
= & -\left\{\left\{\lambda_{1}\left(\xi^{\prime}\right), \lambda_{2}(\eta)\right\}, \lambda_{1}(\xi)\right\}-\left\{\left\{\lambda_{2}(\eta), \lambda_{1}(\xi)\right\}, \lambda_{1}\left(\xi^{\prime}\right)\right\} \\
& +\lambda_{1}\left(\circ_{\eta}\left[\xi, \xi^{\prime}\right]\right) \\
= & -\left\{-\lambda_{1}\left(\circ_{\eta} \xi^{\prime}\right)+c\left(\xi^{\prime}, \eta\right), \lambda_{1}(\xi)\right\} \\
& +\left\{-\lambda_{1}\left(\circ_{\eta} \xi\right)+c(\xi, \eta), \lambda_{1}\left(\xi^{\prime}\right)\right\}+\lambda_{1}\left(\circ_{\eta}\left[\xi, \xi^{\prime}\right]\right) \\
= & \left\{\lambda_{1}\left(\circ_{\eta} \xi^{\prime}\right), \lambda_{1}(\xi)\right\}-\left\{\lambda_{1}\left(\circ_{\eta} \xi\right), \lambda_{1}\left(\xi^{\prime}\right)\right\}+\lambda_{1}\left(\circ_{\eta}\left[\xi, \xi^{\prime}\right]\right) \\
= & \lambda_{1}\left(\left[\circ_{\eta} \xi^{\prime}, \xi\right]\right)-\lambda_{1}\left(\left[\dot{\circ}_{\eta} \xi, \xi^{\prime}\right]\right)+\lambda_{1}\left(\circ_{\eta}\left[\xi, \xi^{\prime}\right]\right) \\
= & 0
\end{aligned}
$$

by using the Jacobi identity of Poisson bracket and the facts that $\lambda_{1}$ is a Lie algebra homomorphism and that $\stackrel{\circ}{\eta}_{\eta}$ is a derivation of $g_{1}$. Since we assumed that $H^{1}\left(g_{1} ; \mathbf{R}\right)$ $=0$, that is, $\left[g_{1}, g_{1}\right]=g_{1}$, we have $c(\xi, \eta)=0$ for every $(\xi, \eta) \in g_{1} \times g_{2}$. Therefore $\nu=0$ identically, which means that $\hat{J}$ is a Lie algebra homomorphic lift of $\rho$. From the lemma we have completed the proof of the theorem.

ACKNOWLEDGEMENT. The author would like to thank the referee for his helpful suggestions which brought improvement to the presentation of this paper. The author also expresses his thanks to Professors Y. Hatakeyama, H. Kitahara and S. Yorozu for their gentle proddings.

\section{REFERENCES}

1. R. Abraham and J. E. Marsden, Foundations of mechanics, Benjamin, New York, 1978.

2. G.-M. Marle, Symplectic manifolds, dynamical groups and Hamiltonian mechanics, Differential Geometry and Relativity, Reidel, Dordrecht, 1976, pp. 249-269.

3. J.-M. Souriau, Structure des systèmes dynamiques, Dunod, Paris, 1970.

4. N. R. Wallach, Symplectic geometry and Fourier analysis, Math. Sci. Press, 1977.

5. A. Weinstein, Lectures on symplectic manifolds, CBMS Regional Conf. Ser. in Math., no. 29, Amer. Math. Soc., Providence, R.I., 1977.

Departmient of Mathematics, Artta University, Aktta 010, Japan 